OPEN ACCESS

Edited by:

Xin Cheng,

Fudan University, China

Reviewed by:

Susanne Wegener

University of Zurich, Switzerland

Anil Man Tuladhar,

Radboud University Nijmegen Medical

Centre, Netherlands

*Correspondence:

Joanna M. Wardlaw

joanna.wardlaw@ed.ac.uk

Specialty section:

This article was submitted to

Stroke,

a section of the journal

Frontiers in Neurology

Received: 30 December 2020

Accepted: 01 April 2021

Published: 04 May 2021

Citation:

Stewart CR, Stringer MS, Shi Y,

Thrippleton MJ and Wardlaw JM (2021) Associations Between White

Matter Hyperintensity Burden, Cerebral Blood Flow and Transit Time in Small Vessel Disease: An Updated

Meta-Analysis.

Front. Neurol. 12:647848

doi: 10.3389/fneur.2021.647848

\section{Associations Between White Matter Hyperintensity Burden, Cerebral Blood Flow and Transit Time in Small Vessel Disease: An Updated Meta-Analysis}

\author{
Catriona R. Stewart ${ }^{1}$, Michael S. Stringer ${ }^{1,2}$, Yulu Shi $^{3}$, Michael J. Thrippleton ${ }^{1,2}$ and \\ Joanna M. Wardlaw ${ }^{1,2 *}$
}

${ }^{1}$ Centre for Clinical Brain Sciences, University of Edinburgh, Edinburgh, United Kingdom, ${ }^{2}$ UK Dementia Research Institute at the University of Edinburgh, Edinburgh Medical School, Edinburgh, United Kingdom, ${ }^{3}$ Beijing Tian Tan Hospital Affiliated to Capital Medical University, Beijing, China

Cerebral small vessel disease (SVD) is a major contributor to stroke and dementia, characterized by white matter hyperintensities $(\mathrm{WMH})$ on neuroimaging. $\mathrm{WMH}$ are associated with reduced cerebral blood flow (CBF) cross-sectionally, though longitudinal associations remain unclear. We updated a 2016 systematic review, identifying 30 new studies, 27 cross-sectional $(n=2,956)$ and 3 longitudinal $(n=440)$. Cross-sectionally, $10 / 27$ new studies $(n=1,019)$ included sufficient data for meta-analysis, which we meta-analyzed with 24 previously reported studies ( $n=1,161)$, total 34 ( $n=2,180)$. Our meta-analysis showed that patients with lower CBF had worse $\mathrm{WMH}$ burden (mean global CBF: standardized mean difference (SMD): -0.45 , 95\% confidence interval (Cl): -0.64, -0.27). Longitudinally, associations between baseline CBF and WMH progression varied: the largest study (5 years, $n=252$ ) found no associations, while another small study ( 4.5 years, $n=52$ ) found that low CBF in the periventricular WMH penumbra predicted WMH progression. We could not meta-analyse longitudinal studies due to different statistical and methodological approaches. We found that CBF was lower in $\mathrm{WMH}$ than in normal-appearing white matter in an additional meta-analysis (5 cross-sectional studies; $n=295$; SMD: -1.51 , 95\% Cl: $-1.94,-1.07$ ). These findings highlight that relationships between resting $\mathrm{CBF}$ and $\mathrm{WMH}$ are complex. Further longitudinal studies analyzing regional $\mathrm{CBF}$ and subsequent $\mathrm{WMH}$ change are required to determine the role of CBF in SVD progression.

\footnotetext{
Keywords: cerebral blood flow, cerebral small vessel disease, stroke, white matter hyperintensities, systematic review, meta-analysis
}

\section{INTRODUCTION}

Cerebral small vessel disease (SVD) develops from pathological changes in the blood vessels supplying the brain and is an underlying cause of about $20 \%$ of all strokes and the most substantial contributor to vascular dementia (1). Advances in neuroimaging have been key in identifying and elucidating common features of SVD, including white matter hyperintensities (WMH), although 
much remains unknown about the underlying pathological mechanisms and there are currently no effective treatments. Vascular changes may occur before WMH are detectable, and are thought to contribute to $\mathrm{WMH}$ development (2). However, whether $\mathrm{CBF}$ reductions precede or follow $\mathrm{WMH}$ progression remains controversial, as hypoperfusion could be a consequence of WMH rather than the cause (3). WMH can be reversible, therefore establishing initial processes involved in WMH development may help identify targets to prevent SVD progression and related pathology $(4,5)$.

Our previous systematic review and meta-analysis of $\mathrm{WMH}$ and $\mathrm{CBF}$ found that more severe $\mathrm{WMH}$ burden was associated with lower $\mathrm{CBF}$, although this association weakened when studies of patients with dementia and poor age matching of controls were excluded (3). Age, dementia, and other vascular risk factors contribute to $\mathrm{CBF}$ reductions and can confound the relationship between WMH burden and CBF (3). Additionally, there were few data on sub-regional brain tissue CBF and longitudinal WMH changes in our previous review (3), as most studies only examined whole brain and/or cortical CBF.

Since 2016, several studies have been published that provide data on regional or tissue-specific $\mathrm{CBF}$ in $\mathrm{WMH}$ and normal appearing white matter (NAWM) cross-sectionally (6-10) as well as longitudinal studies, yet knowledge gaps remain regarding the association between CBF and WMH. Therefore, we updated our previous systematic review and meta-analysis (3) to identify whether these gaps in the literature and previous inconsistencies have been resolved.

\section{MATERIALS AND METHODS}

We updated our previous systematic review by Shi et al. (3), covering from 1946 until December 2015, by conducting a literature search of MEDLINE and EMBASE from 1st January 2016 up to 1st February 2020, using OVID. The search strategy and methodological approach including quality assessment and data extraction were as published by Shi et al. (3), combining exploded search terms relating to small vessel disease and cerebral blood flow with AND (Supplementary Figure 1). We identified additional records by hand-searching, from January 2016 to February 2020, the Journal of Cerebral Blood Flow and Metabolism and within the subject of Cerebrovascular Disease and Stroke in Stroke.

\section{Eligibility Criteria}

We included longitudinal and cross-sectional studies investigating associations between $\mathrm{CBF}$ or transit time metrics, including arterial transit time (ATT) and mean transit time (MTT), and WMH in patients with SVD, as well as studies measuring $\mathrm{CBF}$ velocity (CBFv) using ultrasound Doppler. We included studies using magnetic resonance imaging (MRI), including with phase contrast, arterial spin labeling (ASL) or dynamic susceptibility contrast, positron emission tomography (PET), single-photon computed tomography and computed tomography (CT) perfusion assessment. We excluded studies in pediatric or animal populations, duplicates, conference abstracts and cross-sectional studies lacking analysable data on CBF.

\section{Data Extraction and Analysis}

We screened all potentially eligible publications and extracted standardized data from studies meeting the eligibility criteria. We extracted data on the study population cohort, the type of study, SVD characteristics, measurements, and units of CBF reported. Where available, we extracted data on separate regional $\mathrm{CBF}$ measurements for WMH, defined using MRI or CT, and NAWM, as this was previously identified as a key gap in the literature (3). Where reported we also extracted data on associations between ATT or MTT and WMH. We assessed the quality of each included study using a checklist based on the Strengthening the Reporting of the Observational studies in Epidemiology (STROBE) criteria (11).

We extracted mean and standard deviation (SD) CBF values from disease and control groups or according to WMH burden, where available from text, tables, graphs or, if necessary, Supplementary Material for cross-sectional studies. We recorded associations with WMH burden either alone or as a component of total SVD burden, such as the ordinal SVD score which includes a point for central or cerebral atrophy, lacunes and white matter hyperintensities when rated from CT scans (12) with cerebral microbleeds also included for MRI (13). We included cross-sectional studies with only qualitative data or association analysis on CBF and SVD in the review but not the meta-analysis. We also extracted association analysis data from studies which performed association analysis in addition to providing quantitative data. We extracted information from longitudinal studies on time to follow-up, baseline and follow-up measurements of $\mathrm{CBF}$ and $\mathrm{WMH}$, and their associations. Where unavailable in the published materials, we contacted authors to request unpublished data on baseline and follow-up CBF and/or WMH volume.

\section{Meta-Analysis}

We included all studies reporting mean and SD values for $\mathrm{CBF}$ in patient groups by WMH burden in the cross-sectional meta-analysis. In studies with more than two patient groups of WMH severity, we took mean values to allow for a comparison between patients with negative-to-mild and moderate-to-severe WMH burden following a previously described procedure (3). We extracted $\mathrm{CBF}$ values for gray and white matter in various brain regions, as reported by individual studies. We calculated standardized mean differences (SMDs) and 95\% confidence intervals (CIs) with a random effects model and analyzed new data along with the data reported in our previous review (3). We also extracted CBF values where available from $\mathrm{WMH}$ and NAWM to perform an additional meta-analysis on regional variability in CBF in different white matter tissues. Where studies reported CBF values separately for periventricular $\mathrm{WMH}$ (PVWMH) and deep WMH (DWMH), we took mean values to give a measurement of $\mathrm{CBF}$ in $\mathrm{WMH}$. We used Cochrane Collaboration's Review Manager (RevMan, RRID:SCR_003581, version 5.4) to perform the meta-analyses.

\section{RESULTS}

We identified 783 articles published between January 2016 and February 2020 from the literature search, after removing 
duplicates (Figure 1). Of these, we identified 149 of potential relevance and screened full texts to assess eligibility, 119 were excluded primarily due to lack of analyzable data (45 studies), no data on the association between WMH and CBF (38 studies) and conference abstracts (33 studies). Overall, we included 30 studies for qualitative analysis, of which 15 studies provided data for

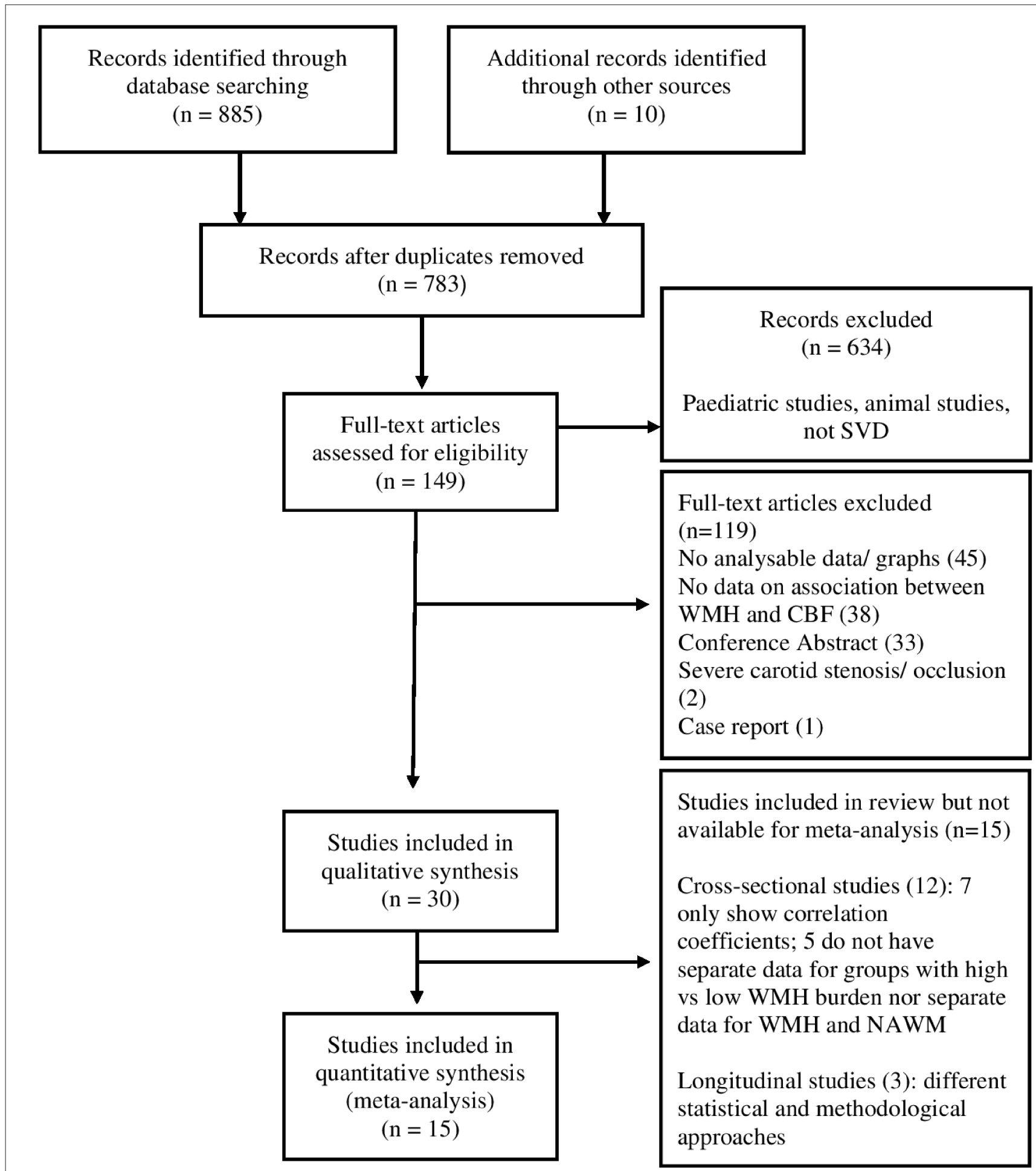

FIGURE 1 | PRISMA flow diagram of literature search. 
meta-analysis (Figure 1). Of these 15, 10 cross-sectional studies were meta-analyzed with data previously extracted from 24 crosssectional studies published between 1946 and 2015 (3) while 5 studies were included in the additional meta-analysis of regional variability in CBF within WMH and NAWM.

Some publications used the same study population cohort: Promjunyakul et al. $(14,15)$ from the Layton Aging and Alzheimer's Disease Center longitudinal aging study and Turk et al. $(16,17)$ with the same cohort of patients with ischemic leukoaraiosis (ILA) and age, sex and risk-factor matched controls without ILA. We ensured that each participant was only included once in any single analysis.

\section{Characteristics of Included Studies}

We extracted data from 27 new cross-sectional $(n=2,956)$ and 3 new longitudinal studies $(n=440)$. All the new studies investigated patients or controls with varying degrees of WMH burden. The characteristics of these 30 studies $(n=3,396)$ are described in Table 1. The average study quality for all identified studies according to the STROBE criteria was 6.5/9 (Supplementary Figure 2). Main reasons for lower overall study quality scores included studies not reporting the number of participants who dropped out (11/30), number and expertise of $\mathrm{WMH}$ assessors (13/30) and a lack of blinding to imaging or clinical data $(14 / 30)$.

Seven of the 27 newly identified cross-sectional studies investigated patients with cognitive impairment or dementia; including Alzheimer's disease (AD) $(20,23)$ mild cognitive impairment $(\mathrm{MCI})(8,10,22)$ and vascular cognitive impairment (VCI) $(9,21)$.

Thirteen of the 27 added cross-sectional studies investigated patients without cognitive impairment including patients with varying degrees of WMH burden $(7,16,24,32)$, hypertensive patients with and without SVD $(6,25,29)$, individuals with Cerebral Autosomal Dominant Arteriopathy with Sub-cortical Infarcts and Leukoencephalopathy (CADASIL) (28), cognitively normal older subjects $(14,27)$ and patients with ischemic stroke (31). Two of these 13 studies investigated the effect of blood pressure reduction on $\mathrm{CBF}$ in SVD, from which only baseline data on $\mathrm{CBF}$ and $\mathrm{WMH}$ burden, prior to any treatment, were extracted $(26,30)$.

Seven of the 27 new cross-sectional studies reported only the results of association analyses and did not provide quantitative data for $\mathrm{CBF}$ or $\mathrm{WMH}$ measurements. Patient groups in these studies included patients with acute stroke (12, 33), MCI (34), vascular depression (35), individuals at varying risk of cerebrovascular disease (36), and older individuals with $\mathrm{WMH}$ $(17,37)$.

The 3 newly identified longitudinal studies $(n=440)$ investigated baseline $\mathrm{CBF}$ and $\mathrm{WMH}$ progression in older individuals with WMH (Table 1), over follow-up periods ranging from two (18) to five (19) years. While 2 studies investigated associations between baseline $\mathrm{WMH}$ volume and change in either subregional (15) or global (19) CBF, one study considered associations between change in $\mathrm{WMH}$ volume and change in cortical gray matter CBF (18).
Fifteen studies used ASL $(6-8,10,14,15,18,22,23,27,29$, 30, 34, 36, 37), 3 used CT perfusion $(20,26,31), 2$ used dynamic susceptibility contrast (DSC) MRI $(9,19)$, one study each used SPECT (21), phase contrast MRI (24), CT angiography/digital subtraction angiography (33), PET (32), and a combination of CT and DSC MRI (12). Five studies used transcranial Doppler ultrasound $(16,17,25,28,35)$.

\section{Cross-Sectional CBF and WMH Association}

We extracted association analysis data from 11 of the new crosssectional studies overall, including the 7 studies which reported association analyses without providing quantitative data and 4 studies which performed association analyses in addition to providing quantitative data $(6,20,27,31)$. Only 5 of these 11 studies adjusted for key vascular risk factors, including hypertension and smoking $(12,17,33,35,36)$.

\section{Cross-Sectional Association Analysis of Global or Regional CBF and WMH}

Four of the new studies analyzed associations between global or regional $\mathrm{CBF}$ and $\mathrm{WMH}$ burden (Table 2). All of these studies found that patients with a worse WMH burden had lower CBF than those with a less severe WMH burden $(20,31,33,34)$, one study showed that worse PVWMH, DWMH and whole-brain $\mathrm{WMH}$ each showed a trend to be associated with lower CBF both in patients with $\mathrm{AD}$ and controls without $\mathrm{AD}(n=203)$ (20). Worse WMH were associated with poorer cerebral collateral circulation, defined as cortical CBF at the outer surface of the brain, in one study which investigated patients with acute stroke $(n=178)$ (33).

Two of the new studies showed that lower mean blood flow velocity $(\mathrm{MBFv})$ in the internal carotid artery and middle cerebral artery were associated with worse WMH burden in patients with ischemic leukoaraiosis $(n=96)(17)$ and vascular depression $(n$ = 76) (35) (Table 2).

\section{Cross-Sectional Association Analysis of SVD Burden and Transit Time}

Arba et al. (12) assessed SVD burden on CT or MRI, using an SVD score and four transit time metrics (MTT, time to maximum flow, time to peak, and arrival time fitted) derived from perfusion CT or DSC MRI, in the asymptomatic cerebral hemisphere of patients with acute ischemic stroke $(n=115)$. Worse SVD scores were associated with more prolonged transit times in white matter of the asymptomatic cerebral hemisphere (i.e., contralateral to the acute ischemic stroke, Table 2). SVD burden was also associated with reduced CBF in the contralateral hemisphere.

\section{Cross-Sectional Association Analysis of CBF in WMH and NAWM}

Three recently published studies found that CBF was lower within WMH than in NAWM (Table 3). Higher WMH volume in older patients with hypertension $(n=185)(6)$ was associated with lower $\mathrm{CBF}$ within WMH. However, no clear relationship 
TABLE 1 | Characteristics of all studies included in this updated systematic review and meta-analysis.

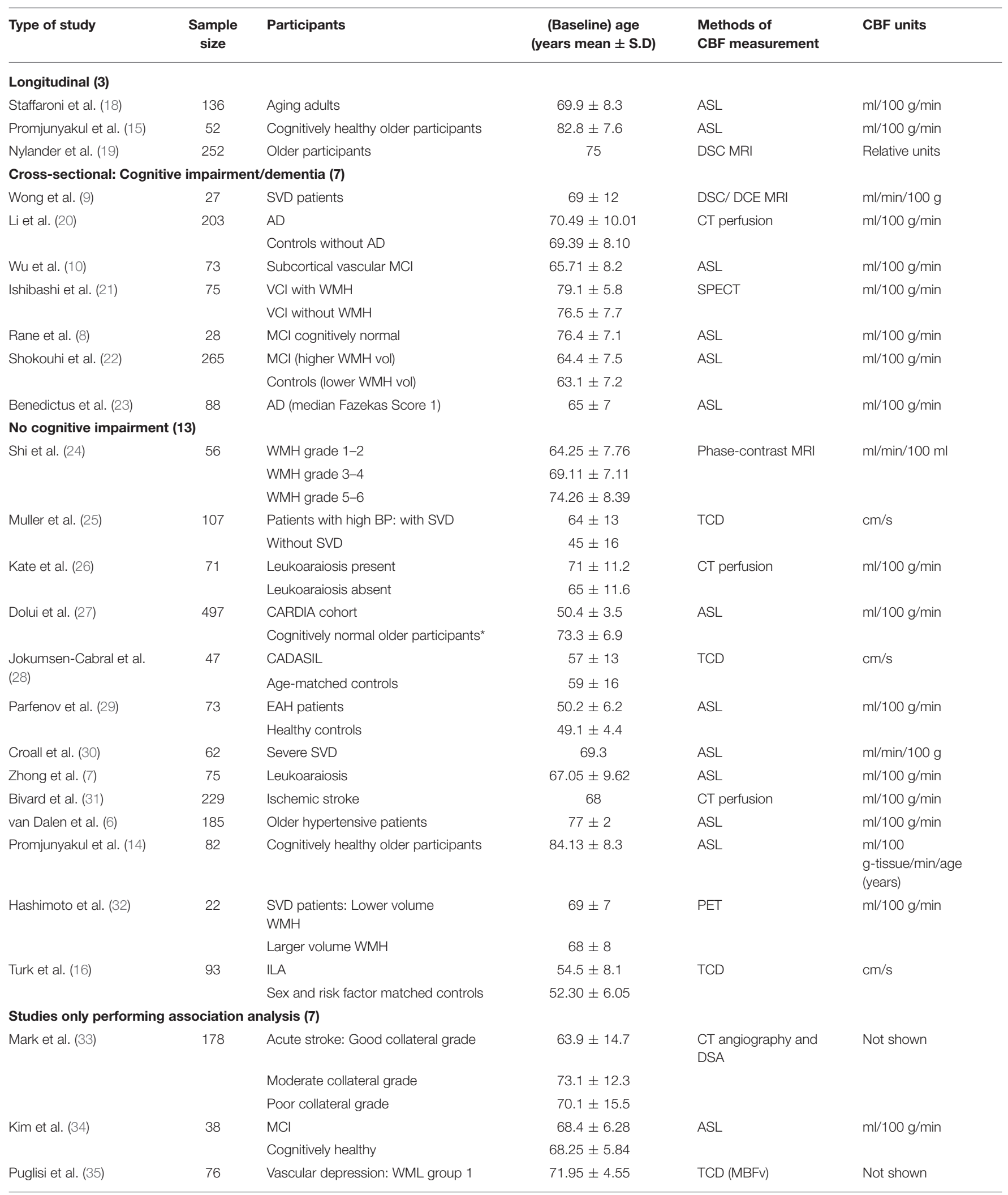


TABLE 1 | Continued

\begin{tabular}{|c|c|c|c|c|c|}
\hline Type of study & $\begin{array}{l}\text { Sample } \\
\text { size }\end{array}$ & Participants & $\begin{array}{c}\text { (Baseline) age } \\
\text { (years mean } \pm \text { S.D) }\end{array}$ & $\begin{array}{l}\text { Methods of } \\
\text { CBF measurement }\end{array}$ & CBF units \\
\hline & & WML group 2 & $71.81 \pm 4.71$ & & \\
\hline & & WML group 3 & $73.96 \pm 6.71$ & & \\
\hline \multirow[t]{2}{*}{ Bahrani et al. (36) } & 26 & High-risk CVD & $83 \pm 4.6$ & ASL & Relative units \\
\hline & & Low-risk CVD & $72.7 \pm 4.2$ & & \\
\hline Shi et al. (37) & 69 & Older participants with WMH & $70.78 \pm 3.94$ & ASL & Relative units \\
\hline Arba et al. (12) & 115 & Acute ischemic stroke & 81 & CT perfusion/ DSC MRI & Not shown \\
\hline \multirow[t]{2}{*}{ Turk et al. (17) } & 96 & ILA & $54.90 \pm 8.27$ & Ultrasound doppler & $\mathrm{mm}^{3} / \mathrm{s}$ \\
\hline & & $\begin{array}{l}\text { Age, sex and risk-factor matched } \\
\text { controls }\end{array}$ & $52.39 \pm 7.34$ & & \\
\hline
\end{tabular}

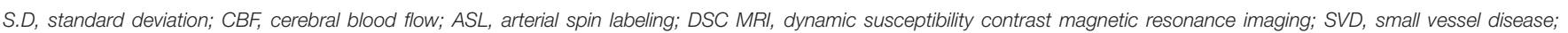

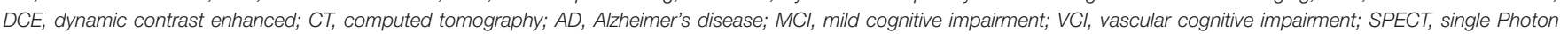

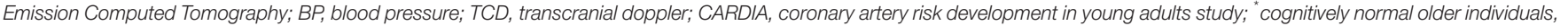

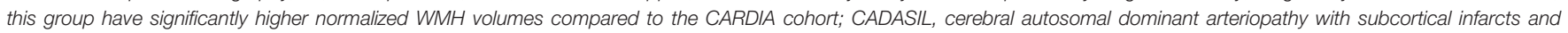

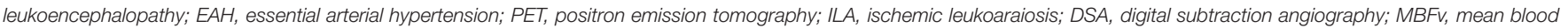
flow velocity; WML, white matter lesion; CVD, cerebrovascular disease.

was seen between WMH volume and CBF in NAWM or gray matter (Table 3). Cognitively healthy older participants and healthy middle-aged participants $(n=497)$ showed significantly lower CBF within WMH compared to normal appearing white matter (27). Similarly, in one small study $(n=26)$, CBF was lower within DWMH and PVWMH compared to NAWM (36). In contrast, one exploratory study (37) in healthy older participants $(n=69)$ found in white matter regions where most patients had WMH; higher WMH burden correlated with higher gray matter perfusion in the opposite cerebral hemisphere.

\section{Sensitivity Analyses of Age, Dementia, and Key Vascular Risk Factors}

We further refined the cross-sectional association analyses by removing studies investigating patients with dementia, studies without age-matching and studies which did not adjust for key vascular risk factors. The direction of effect in the remaining studies did not change: lower blood flow velocity $(17,35)$ and prolonged perfusion CT/DSC transit time metrics (12) remained associated with $\mathrm{WMH}$, as well as lower $\mathrm{CBF}$ within WMH compared to NAWM (36). Only one cross-sectional study investigated $\mathrm{WMH}$ in association with global or regional CBF while adjusting for key vascular risk factors, showing worse WMH was associated with poorer collateral blood flow (33).

\section{Longitudinal Analyses of CBF and WMH}

The largest of the three new longitudinal studies (19) in a cohort of 406 randomly selected 75-year-old participants at baseline and 252 at follow-up, found no significant association between baseline CBF with $\mathrm{WMH}$ progression at a five-year follow-up when adjusting for baseline $\mathrm{WMH}$ and sex (Table 4). In 136 functionally normal older individuals, Staffaroni et al. (18) found that longitudinal reductions in global CBF were associated with increasing WMH burden, adjusting for age, sex and education. Promjunyakul et al. (15), in 52 cognitively healthy older participants, found that lower baseline CBF within the
PVWMH penumbra was associated with WMH growth at followup, while reduced white matter perfusion remained predictive of PVWMH but not DWMH progression, adjusting for age and sex. These 440 patients from the three recent longitudinal studies add to the 1,079 patients in 4 studies included in our 2016 review (3). Summarizing all 7 longitudinal studies $(n=$ 1,519 ), the two largest studies (total $n=827,575+252$ ) found low baseline CBF did not predict worsening WMH; one previous and one new study $(n=390+136)$ found low CBF predicted increased PVWMH but not deep WMH progression or predicted global WMH; the two smallest studies $(n=40$ and 52) found low $\mathrm{CBF}$ in NAWM/penumbral tissue predated $\mathrm{WMH}$ growth at follow-up; and one prior study $(n=74)$ found $\mathrm{CBF}$ increased in some regions and decreased in other regions as WMH progressed.

Longitudinal meta-analysis was not possible due to insufficient data and different statistical and methodological approaches between studies. The two studies with follow-up CBF measures $(18,38)$ carried out their statistics differently and therefore could not be combined.

\section{Cross-Sectional Meta-Analysis of CBF and WMH Burden}

We included 34 cross-sectional studies $(n=2,180)$ in this updated meta-analysis, of which 24 had been previously identified in our 2016 review (3). The 10 newly identified studies covered 4 brain regions, including mean global CBF $(20,21,24$, $29)$, mean basal ganglia blood flow (32), total gray matter $(6,22)$ and centrum semiovale (32) (Figure 2A). We extracted data on $\mathrm{CBF}$ velocity, as measured from the middle cerebral artery (vMCA) $(16,25,28)$ and included this under a new subgroup in the meta-analysis. We found no additional published data for 7 brain regions included in our previous review (3): four in gray matter (frontal, temporal, parietal, and occipital) and three in white matter (total, frontal, and occipital). 
TABLE 2 | Association analysis of (A) global or regional CBF with WMH; (B) mean blood flow velocity with WMH and (C) transit time with SVD burden.

\begin{tabular}{|c|c|c|c|c|c|c|}
\hline Study & $\begin{array}{l}\text { Sample } \\
\text { size }\end{array}$ & Statistical method & $\begin{array}{l}\text { Results } \\
\text { Variables }\end{array}$ & Coefficients & $P$-values & $\begin{array}{l}\text { Adjusted for other } \\
\text { variables }\end{array}$ \\
\hline \multicolumn{7}{|c|}{ (A) Global or regional CBF association with WMH } \\
\hline Bivard et al. (31) & 229 & Linear regression model & $\begin{array}{l}\text { Hypoperfusion and } \\
\text { likelihood of WMH }\end{array}$ & $\mathrm{OR}=7.61$ & $<0.001$ & No \\
\hline \multirow[t]{4}{*}{ Kim et al. (34) } & 38 & Linear regression analysis & $\begin{array}{l}\text { Mean parietal lobe CBF and } \\
\text { WMH volume }\end{array}$ & $t(15)=-3$ & 0.009 & Gender \\
\hline & & & $\begin{array}{l}\text { Mean temporal lobe CBF } \\
\text { and } \mathrm{WMH} \text { volume }\end{array}$ & $t(15)=-3.89$ & 0.001 & \\
\hline & & & $\begin{array}{l}\text { Mean occipital lobe CBF } \\
\text { and } \mathrm{WMH} \text { volume }\end{array}$ & $t(15)=-4.71$ & 0.001 & \\
\hline & & & $\begin{array}{l}\text { Mean frontal lobe CBF and } \\
\text { WMH volume }\end{array}$ & $t(15)=-2.58$ & 0.021 & \\
\hline \multirow[t]{6}{*}{ Li et al. (20) } & 203 & $\begin{array}{l}\text { Spearman correlation } \\
\text { analysis }\end{array}$ & $\begin{array}{l}\text { Whole-brain WMH severity } \\
\text { and CBF (AD patients) }\end{array}$ & $r=-0.162$ & 0.068 & Not shown \\
\hline & & & $\begin{array}{l}\text { PVWMH severity and CBF } \\
\text { (AD patients) }\end{array}$ & $r=-0.219$ & 0.013 & \\
\hline & & & $\begin{array}{l}\text { DWMH severity and CBF } \\
\text { (AD patients) }\end{array}$ & $r=-0.099$ & 0.268 & \\
\hline & & & $\begin{array}{l}\text { Whole-brain WMH severity } \\
\text { and CBF (controls) }\end{array}$ & $r=-0.034$ & 0.071 & \\
\hline & & & $\begin{array}{l}\text { PVWMH severity and CBF } \\
\text { (controls) }\end{array}$ & $r=-0.071$ & 0.546 & \\
\hline & & & $\begin{array}{l}\text { DWMH severity and CBF } \\
\text { (controls) }\end{array}$ & $r=-0.003$ & 0.978 & \\
\hline \multirow[t]{3}{*}{ Mark et al. (33) } & 178 & $\begin{array}{l}\text { Multivariate logistic } \\
\text { regression analysis }\end{array}$ & $\begin{array}{l}\text { PVWM Fazekas score and } \\
\text { collateral flow }\end{array}$ & NA & $<0.0001$ & \multirow{3}{*}{$\begin{array}{l}\text { Age, gender, hypertension, } \\
\text { hyperlipidemia coronary } \\
\text { artery disease, atrial } \\
\text { fibrillation, congestive heart } \\
\text { failure and smoking history }\end{array}$} \\
\hline & & & $\begin{array}{l}\text { DWM Fazekas score and } \\
\text { collateral flow }\end{array}$ & NA & $<0.0001$ & \\
\hline & & & $\begin{array}{l}\text { Total Fazekas score and } \\
\text { collateral flow }\end{array}$ & NA & $<0.0001$ & \\
\hline \multicolumn{7}{|c|}{ (B) Mean blood flow velocity association with WMH } \\
\hline \multirow[t]{2}{*}{ Turk et al. (17) } & 96 & $\begin{array}{l}\text { Spearman's correlation } \\
\text { coefficient }\end{array}$ & $\begin{array}{l}\text { Correlation of average mean } \\
\text { blood flow in ICA }\left(\mathrm{mm}^{3} / \mathrm{s}\right) \\
\text { with ILA }\end{array}$ & Spearman's $p=-0.232$ & 0.025 & $\begin{array}{l}\text { Age, gender, and CVD risk } \\
\text { factors }\end{array}$ \\
\hline & & Logistic regression & $\begin{array}{l}\text { Association of average } \\
\text { mean blood flow in ICA } \\
\left(\mathrm{mm}^{3} / \mathrm{s}\right) \text { with ILA }\end{array}$ & $\mathrm{OR}=0.577(0.368-0.902)$ & 0.016 & \\
\hline Puglisi et al. (35) & 76 & Spearman correlation & $\begin{array}{l}\text { Correlation between MBFv } \\
\text { in MCA and } \mathrm{WMH} \text { severity }\end{array}$ & $r=-0.34$ & 0.002 & $\begin{array}{l}\text { Demographic variables and } \\
\text { clinical variables (including } \\
\text { vascular risk factors) }\end{array}$ \\
\hline \multicolumn{7}{|c|}{ (C) Transit time association with SVD burden } \\
\hline \multirow[t]{4}{*}{ Arba et al. (12) } & 115 & Logistic regression model & $\begin{array}{l}\text { SVD score and mean transit } \\
\text { time }\end{array}$ & $\mathrm{OR}=2.80(1.56-5.03)$ & $<0.05$ & $\begin{array}{l}\text { Age, NIHSS, sex, } \\
\text { hypertension, and diabetes }\end{array}$ \\
\hline & & & $\begin{array}{l}\text { SVD score and time to } \\
\text { maximum flow }\end{array}$ & $\mathrm{OR}=2.36(1.37-4.09)$ & $<0.05$ & \\
\hline & & & SVD score and time to peak & $\mathrm{OR}=1.52(0.93-1.05)$ & N.S. & \\
\hline & & & $\begin{array}{l}\text { SVD score and arrival time } \\
\text { fitted }\end{array}$ & $\mathrm{OR}=3.59(1.92-6.75)$ & $<0.01$ & \\
\hline
\end{tabular}

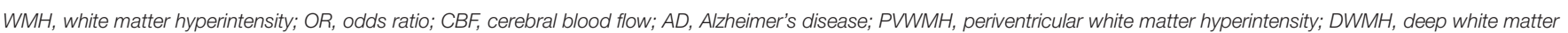

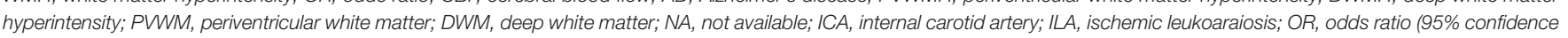

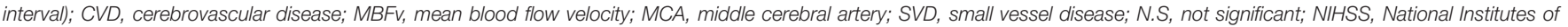
Health Stroke Scale. P-values $<0.05$ are shown in bold.

Of the four regions where additional data was identified, patients with moderate-to-severe WMH burden had lower $\mathrm{CBF}$ than those with negative-to-mild WMH burden in three regions: global CBF (SMD: $-0.45,95 \%$ CI: $-0.64,-0.27$ ), total gray matter (SMD: $-0.37,95 \%$ CI: $-0.69,-0.05$ ) and centrum semiovale (SMD: $-1.25,95 \%$ CI: $-1.96,-0.54$ ) 
TABLE 3 | Association analysis comparing CBF within WMH and NAWM.

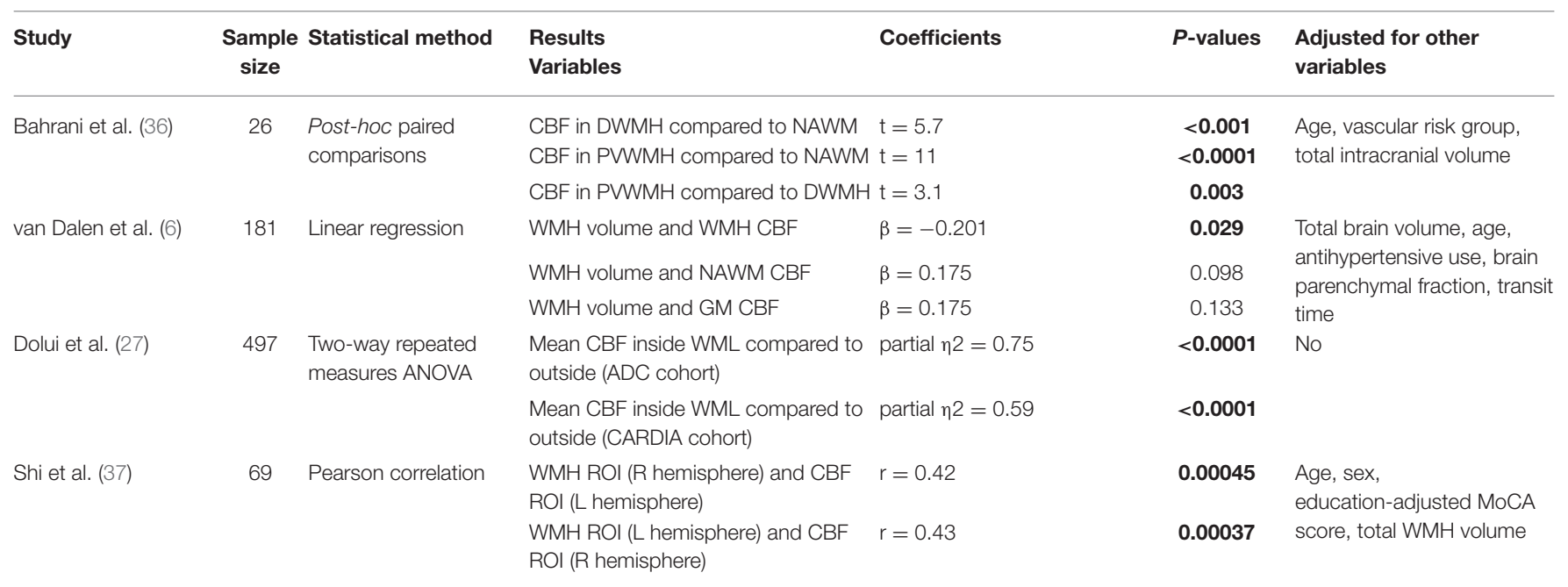

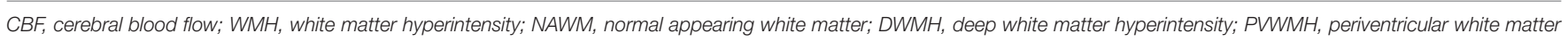

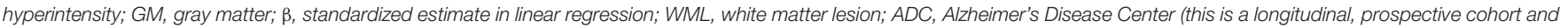

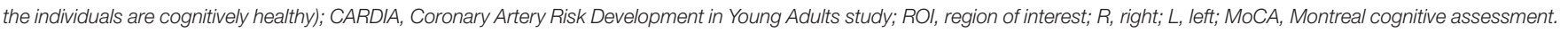
$P$-values $<0.05$ are shown in bold.

TABLE 4 | Results from longitudinal studies.

\begin{tabular}{|c|c|c|c|c|}
\hline & & Nylander et al. (19) & Staffaroni et al. (18) & Promjunyakul et al. (15) \\
\hline Sample size & & 252 & 136 & 52 \\
\hline Follow-up time (years) & & 5 & 2.3 & 4.5 \\
\hline CBF & At baseline & NA & $27.07 \pm 6.38^{*}$ & $\begin{array}{l}\text { WM CBF: } 27.0 \pm 6.6 \\
\text { GM CBF: } 59.3 \pm 10.0 \\
\text { PWWMH CBF: } 14.09 \pm 6.05 \\
\text { DWMH CBF: } 16.12 \pm 6.85\end{array}$ \\
\hline CBF & At follow-up & NA & $24.47 \pm 5.61^{*}$ & NA \\
\hline WMH (ml) & At baseline & $10.5 \pm 5.2(n=406)$ & $3.4 \pm 3.8^{*}$ & $\begin{array}{l}\text { Mean PWWMH vol. }=10.3 \pm 11.7 \\
\text { Mean DWMH vol. }=1.6 \pm 1.2\end{array}$ \\
\hline WMH (ml) & At follow-up & $11.9 \pm 5.7(n=229)$ & $4.6 \pm 4.8$ * & $\begin{array}{l}\text { Mean PWWMH vol. growth }=3.7 \pm 6 \mathrm{ml} \\
\text { Mean DWMH vol. growth }=0.4 \pm 1 \mathrm{ml}\end{array}$ \\
\hline Baseline CBF and baseline WMH volume & $\begin{array}{l}\text { Coefficient } \\
P \text {-value }\end{array}$ & $\begin{array}{l}\text { NA } \\
\text { N.S. }\end{array}$ & $\begin{array}{l}B=-0.08, b=-0.01 \\
0.358\end{array}$ & NA \\
\hline Baseline CBF and $\triangle \mathrm{WMH}$ volume & $\begin{array}{l}\text { Coefficient } \\
P \text {-value }\end{array}$ & $\begin{array}{l}\text { NA } \\
\text { N.S. }\end{array}$ & NA & NA \\
\hline Baseline WMH volume and $\Delta \mathrm{CBF}$ & $\begin{array}{l}\text { Coefficient } \\
P \text {-value }\end{array}$ & NA & NA & NA \\
\hline$\Delta \mathrm{WMH}$ volume and $\Delta \mathrm{CBF}$ & $\begin{array}{l}\text { Coefficient } \\
P \text {-value }\end{array}$ & NA & $\begin{array}{l}b=-0.02 \\
0.007\end{array}$ & NA \\
\hline Adjusted for other variables & & Baseline value, sex & Age, sex, education & Age, sex \\
\hline
\end{tabular}

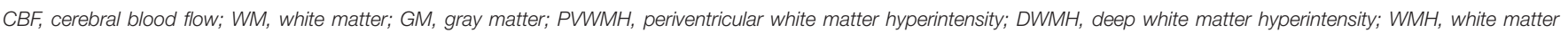

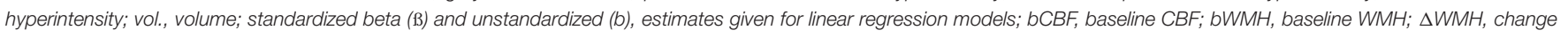
in WMH; $\triangle \mathrm{CBF}$, change in CBF; NA, not available.

*Unpublished data received from author upon request.

(Figure 2B). In the basal ganglia there was no difference in $\mathrm{CBF}$ between patients with moderate-to-severe and negative-to-mild WMH burden (SMD: $-0.86,95 \% \mathrm{CI}:-1.97,0.25$ ). Participants with worse WMH burden had lower blood flow velocities in the middle cerebral artery (SMD: $-0.34,95 \% \mathrm{CI}:-0.64$, $-0.05)$. 


\begin{tabular}{|c|c|c|c|c|c|c|c|c|c|c|c|}
\hline \multirow{3}{*}{$\frac{A_{\text {Study or Subgroup }}}{\text { 1.1.1 mean global CBF }}$} & \multicolumn{3}{|c|}{ moderate to severe WMH } & \multicolumn{3}{|c|}{ negative to mild WMH } & \multirow{2}{*}{\multicolumn{3}{|c|}{ Std. Mean Difference }} & \multirow{2}{*}{\multicolumn{2}{|c|}{$\begin{array}{l}\text { Std. Mean Difference } \\
\text { IV, Random, } 95 \% \mathrm{Cl}\end{array}$}} \\
\hline & Mean & so & Total & Mean & SD & Total & Weight & & 1 Year & & \\
\hline & & & & & & & & & & & \\
\hline Fazekas, F, et al. 1988 & 39.7 & 4.4 & 12 & 42.7 & 3.1 & 11 & $1.3 \%$ & $-0.75[-1.61,0.10]$ & 1988 & -1 & \\
\hline Kimura, M. et al. 2003 & 41.5 & 2.7 & 9 & 47.5 & 5.6 & 11 & $1.1 \%$ & $-1.26[-2.25,-0.28]$ & 2003 & - & \\
\hline Zheng, L. S. et al. 2006 & 369.1 & 41.6 & 20 & 394.2 & 36.5 & 15 & $1.5 \%$ & $-0.62[-1.31,0.07]$ & 2006 & $\rightarrow$ & \\
\hline De Bastos-Leite, A. J. et al. 2008 & 43.5 & 6.3 & 7 & 55.7 & 9.2 & 14 & $1.1 \%$ & $-1.40[-2.42,-0.37]$ & 2008 & - & \\
\hline Kimura. N. et al. 2012 & 37.6 & 3 & 57 & 38.6 & 3.5 & 41 & $1.9 \%$ & $-0.31[-0,71,0.10]$ & 2012 & & \\
\hline Ishibashi, M. et al. 2018 & 38.2 & 3.1 & $\frac{\pi}{46}$ & 39.7 & $\frac{5}{5}$ & 29 & $1.8 \%$ & $-0.38[-0.85,0.09]$ & 2018 & - & \\
\hline Parlenov, V.A. et al. 2018 & 38.85 & 6.3 & 17 & 39.2 & 4.7 & 16 & $1.5 \%$ & $-0.06[-0.74,0.62]$ & 2018 & & \\
\hline Li, R. et al. 2019 & 26.98 & 10.48 & 128 & 31.1 & 9.48 & 75 & $2.0 \%$ & $-0.41[-0.69,-0.12]$ & 2019 & 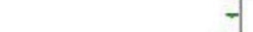 & \\
\hline Shi, Y, et al, 2020 & 58.35 & 8.44 & 32 & 62.62 & 10.65 & 24 & $1.7 \%$ & $-0.45[-0.98,0.09]$ & 2020 & - & \\
\hline$\frac{\text { Subtotal }(95 \% \mathrm{Cl})}{\text { Sula }}$ & 00.05 & 0.44 & 328 & 0.02 & 00.00 & $\frac{24}{236}$ & $\frac{1.10}{13.8 \%}$ & $\frac{-0.45[-0.64,-0.27]}{-0.640}$ & 2020 & 1 & \\
\hline \multicolumn{12}{|c|}{$\begin{array}{l}\text { Heterogeneity: } \mathrm{Tau}^{2}=0.01 ; \mathrm{Ch}^{2}=8.56, \mathrm{df}=8(\mathrm{P}=0.38) ; \mathrm{l}^{2}=6 \% \\
\text { Test for overall effect: } Z=4.88(P<0.00001)\end{array}$} \\
\hline \multicolumn{12}{|l|}{ 1.1.2 mean basal ganglia blood flow } \\
\hline Hatazawa, J. et al. 1997 & 44.9 & 6.9 & 15 & 70.1 & 12 & 8 & $0.9 \%$ & $-2.72[-3.94,-1.50]$ & 1997 & - & \\
\hline Markus, H. S. et al. 2000 & 44.57 & 11.12 & 8 & 52.11 & 16.4 & 9 & $1.1 \%$ & $-0.50[-1.48,0.47]$ & 2000 & $\longrightarrow$ & \\
\hline Nezu, T. et al. 2012 & 47.1 & 9.8 & 9 & 54.6 & 11.3 & 9 & $1.1 \%$ & $-0,68[-1,63,0.28]$ & 2012 & & \\
\hline Hashimoto, T. et al. 2016 & 31.5 & 4.9 & 12 & 30.3 & $\frac{6}{6}$ & 10 & $1.3 \%$ & $0.21-0.63 .1 .05]$ & 2016 & & \\
\hline Subtotal $(95 \% \mathrm{Cl})$ & & & 44 & & & $\frac{736}{36}$ & $4.4 \%$ & $-0.86[-1.97,0.25]$ & & & \\
\hline \multicolumn{12}{|c|}{$\begin{array}{l}\text { Heterogeneity: } \text { Tau }^{2}=1.02 ; \mathrm{Ch}^{2}=15.23 \text {, df }=3(P=0.002) ; 1^{2}=80 \% \\
\text { Test for overall effect: } Z=1.52(P=0.13)\end{array}$} \\
\hline \multicolumn{12}{|l|}{ 1.1.3 total grey matter } \\
\hline Kobayashi, S. et al. 1991 & 66.1 & 16.9 & 28 & 66.6 & 13.8 & 218 & $1.9 \%$ & $-0.04[-0.43,0.36]$ & 1991 & & \\
\hline Oishi, M. et al. 1999 & 46 & 8.17 & 30 & 57.9 & 7.5 & 15 & $1.5 \%$ & $-1.47[-2.17,-0.77]$ & 1999 & - & \\
\hline Markus, H. S. et al. 2000 & 39.76 & 8.38 & 8 & 40.7 & 10.92 & 9 & $1.1 \%$ & $-0.09[-1.04,0.86]$ & 2000 & & \\
\hline O'Sullivan, M. et al. 2002 & 40 & 15.1 & 21 & 48.1 & 17.1 & 15 & $1.5 \%$ & $-0.50[-1.17,0.18]$ & 2002 & -1 & \\
\hline Huynh, T. J. et al 2008 & 27.7 & 6.4 & 9 & 33.9 & 6.9 & 26 & $1.3 \%$ & $-0.89[-1.68,-0.10]$ & 2008 & - & \\
\hline De Bastos-Leite, A. J. et al. 2008 & 57.9 & 8.6 & 7 & 69.9 & 12.6 & 14 & $1.1 \%$ & $-1.00[-1.97,-0.04]$ & 2008 & & \\
\hline Wagner, M. 2015 & 53.06 & 21.85 & 18 & 48.43 & 12.87 & 18 & $1.5 \%$ & $0.25[-0.40,0.91]$ & 2015 & & \\
\hline van Dalen, J.W. et al. 2016 & $\frac{0.03}{42}$ & 15 & 92 & 47 & 14 & 93 & $2.0 \%$ & $-0.34[-0.63,-0.05]$ & 2016 & & \\
\hline Shokouni, M. et al. 2018 & 46.4 & 8.4 & 185 & 45.7 & 8.6 & & $2.1 \%$ & & 2018 & & \\
\hline Subtotal $(95 \% \mathrm{Cl})$ & 30.4 & 0,4 & 398 & $4+1.1$ & 0.0 & $\frac{508}{488}$ & $\frac{2.10}{14.1 \%}$ & $-0.37[-0.69,-0.05]$ & 20000 & 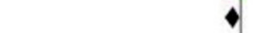 & \\
\hline $\begin{array}{l}\text { Heterogeneity: } \operatorname{Tau}^{2}=0.15 ; \mathrm{Chi}^{2}= \\
\text { Test for overall effect: } Z=2.25(\mathrm{P}=\end{array}$ & $\begin{array}{l}7.01, d f=8 \\
0.02)\end{array}$ & $P=0.000$ & $1^{2}=70^{\circ}$ & & & & & & & & \\
\hline 1.1.4 frontal grey matter & & & & & & & & & & & \\
\hline Kobari, M. et al. 1990 & 36.97 & 6.97 & 16 & 43.03 & 15.15 & 15 & $1.4 \%$ & $-0.51[-1.22,0.21]$ & 1990 & - & \\
\hline Kawamura, J. et al. 1993 & 38.89 & 6.67 & 23 & 52.59 & 8.15 & 16 & $1.4 \%$ & $-1.84[-2.61,-1.07]$ & 1993 & - & \\
\hline Kuwabara, Y. et al. 1996 & 38.6 & 5.9 & 5 & 37.1 & 3.7 & 13 & $1.0 \%$ & $0.33[-0.71,1.37]$ & 1996 & & \\
\hline Yamaji, S. et al. 1997 & 48.7 & 8.4 & 16 & 52 & 6.5 & 16 & $1.5 \%$ & $-0.43[-1.13,0.27]$ & 1997 & -7 r a r a & \\
\hline Ibayashi, S. et al. 2000 & 22.5 & 3.06 & 6 & 32.5 & 5.64 & 9 & $0.8 \%$ & $-1.96[-3.27,-0.64]$ & 2000 & - & \\
\hline Yao, H. et al. 2000 & 42.7 & 4.1 & 5 & 44 & 7.1 & 5 & $0.9 \%$ & $-0.20[-1.45,1.04]$ & 2000 & $\rightarrow$ & \\
\hline O'Sullivan, M. et al. 2002 & 38.9 & 16.4 & 21 & 48.1 & 15.9 & 15 & $1.5 \%$ & $-0.56[-1.23,0.12]$ & 2002 & - & \\
\hline Schuff, N. et al. 2009 & 38.9 & 14.4 & 8 & 56.4 & 7.9 & 18 & $1.1 \%$ & $-1.66[-2.62,-0.69]$ & 2009 & - & \\
\hline Nezu, T. et al. 2012 & 36.1 & 7.2 & 9 & 40.2 & 7.3 & 9 & $1.2 \%$ & $-0.54[-1.48,0.41]$ & 2012 & $\rightarrow$ & \\
\hline $\mathrm{Fu}$ J. et al. 2014 & 46.5 & 12.6 & & 50.1 & 10.7 & & $1.7 \%$ & $-0.30[-0.83,0.24]$ & 2014 & -1 & \\
\hline $\begin{array}{l}\text { Fu, et all. } 2014 \\
\text { Subtotal }(95 \% \mathrm{Cl})\end{array}$ & 46.5 & 12.6 & 142 & 50.1 & 10.7 & ${ }_{139}^{23}$ & $12.5 \%$ & $\begin{array}{r}-0.30[-0.0 .83,0.24] \\
-0.73,-0.31]\end{array}$ & 2014 & $\bullet$ & \\
\hline $\begin{array}{l}\text { Heterogeneity: } \text { Tau }^{2}=0.27 ; \mathrm{Chi}^{2}= \\
\text { Test for overall effect: } Z=3.38(P=\end{array}$ & $\begin{array}{l}3.28, d f=s \\
0.0007)\end{array}$ & $P=0.006$ & $P^{2}=61 \%$ & & & & & & & & \\
\hline 1.1.5 temporal grey matter & & & & & & & & & & & \\
\hline Kobari, M. et al. 1990 & 36.97 & 6.97 & 16 & 41.21 & 10.91 & 15 & $1.4 \%$ & $-0.45[-1.17,0.26]$ & 1990 & -7 & \\
\hline Kawamura, J. et al. 1993 & 37.04 & 6.3 & 16 & 50.37 & 5.19 & 23 & $1.3 \%$ & $-2.30[-3.14,-1.47]$ & 1993 & - & \\
\hline Kuwwabara, Y. et al. 1996 & 39.5 & 6.4 & 5 & 38.1 & 4 & 13 & $1.0 \%$ & $0.28[-0.75,1.32]$ & 1996 & & \\
\hline Yamaji, S. et al. 1997 & 41.3 & 4.7 & 16 & 46.1 & 8 & 16 & $1.4 \%$ & $-0.71[-1.43,0.00]$ & 1997 & - & \\
\hline Ibayashi, S. et al. 2000 & 23.75 & 4.61 & 6 & 33.75 & 5.25 & 9 & $0.8 \%$ & $-1.88[-3.17,-0.58]$ & 2000 & - & \\
\hline Schuff, N. et al. 2009 & 37.6 & 12.6 & 8 & 52.7 & 19.1 & 18 & $1.2 \%$ & $-0.84[-1.71,0.03]$ & 2009 & -1 & \\
\hline Fu, J. et al. 2014 & 48.1 & 13 & 33 & 54.7 & 9.2 & 23 & $1.7 \%$ & $-0.56[-1.10,-0.02]$ & 2014 & -1 & \\
\hline Subtotal $(95 \% \mathrm{Cl})$ & & & 100 & & & 117 & $9.0 \%$ & $-0.89[-1.46,-0.31]$ & & $\bullet$ & \\
\hline $\begin{array}{l}\text { Heterogeneity: } \operatorname{Tau}^{2}=0.41 ; \mathrm{Ch}^{2}= \\
\text { Test for overall effect: } \mathrm{Z}=3.03(\mathrm{P}\end{array}$ & $\begin{array}{l}1.00, \mathrm{df}=6 \\
0.002)\end{array}$ & $P=0.002$ & $I^{2}=71 \%$ & & & & & & & & \\
\hline 1.1.6 pariotal grey matter & & & & & & & & & & & \\
\hline Kawamura, J. et al. 1993 & 42.22 & 8.52 & 23 & 51.11 & 9.63 & 16 & $1.5 \%$ & $-0.97[-1.65,-0.29]$ & 1993 & - & \\
\hline Kuwabara, Y. et al. 1996 & 40.2 & 9.5 & 5 & 39.5 & 3.7 & 13 & $1.1 \%$ & $0.12[-0.92,1.15]$ & 1996 & & \\
\hline Yamaji, S. et al. 1997 & 47.5 & $\begin{array}{l}9.3 \\
6.3\end{array}$ & 16 & 48.7 & 5.7 & 16 & $1.5 \%$ & $-0.19[-0.89,0.50]$ & 1997 & - & \\
\hline Ibayashi, S. et al. 2000 & 22.8 & 6.58 & 6 & 33.25 & 4.68 & 9 & $0.8 \%$ & $-1.79[-3.07,-0.52]$ & 2000 & - & \\
\hline O'Sullivan, M. et al. 2002 & 41.8 & 16.2 & 21 & 50.1 & 19.2 & 15 & $1.5 \%$ & $-0.46[-1.14,0.21]$ & 2002 & -7 & \\
\hline Schuff, N. et al. 2009 & 35.5 & 11.7 & 8 & 58.7 & 9.8 & 18 & $1.0 \%$ & $-2.16[-3.21,-1.11]$ & 2009 & - & \\
\hline Nezu, T. et al. 2012 & 39.7 & 4.8 & 9 & 45.7 & 10.5 & 9 & $1.1 \%$ & $-0.70[-1.66,0.26]$ & 2012 & $\rightarrow$ & \\
\hline Fu, J. et al. 2014 & 46.63 & 12.53 & 33 & 50.53 & 12.79 & 23 & $1.7 \%$ & $-0.30[-0.84,0.23]$ & 2014 & - & \\
\hline Subtotal $(95 \% \mathrm{Cl})$ & & & 121 & & & 119 & $10.2 \%$ & $-0.72[-1.17,-0.26]$ & & $\bullet$ & \\
\hline $\begin{array}{l}\text { Heterogeneity: } \text { Tau }^{2}=0.24 ; \mathrm{Chi}^{2}= \\
\text { Test for overall effect: } Z=3.11(\mathrm{P}\end{array}$ & $\begin{array}{l}7.53, \mathrm{df}=7 \\
0.002)\end{array}$ & $P=0.01)$ & $=60 \%$ & & & & & & & & \\
\hline & & & & & & & & & & $-10 \quad \begin{array}{l}-5 \\
\end{array}$ & $\stackrel{5}{5}{ }_{\text {ncreased CBF in SVD }}^{10}$ \\
\hline
\end{tabular}




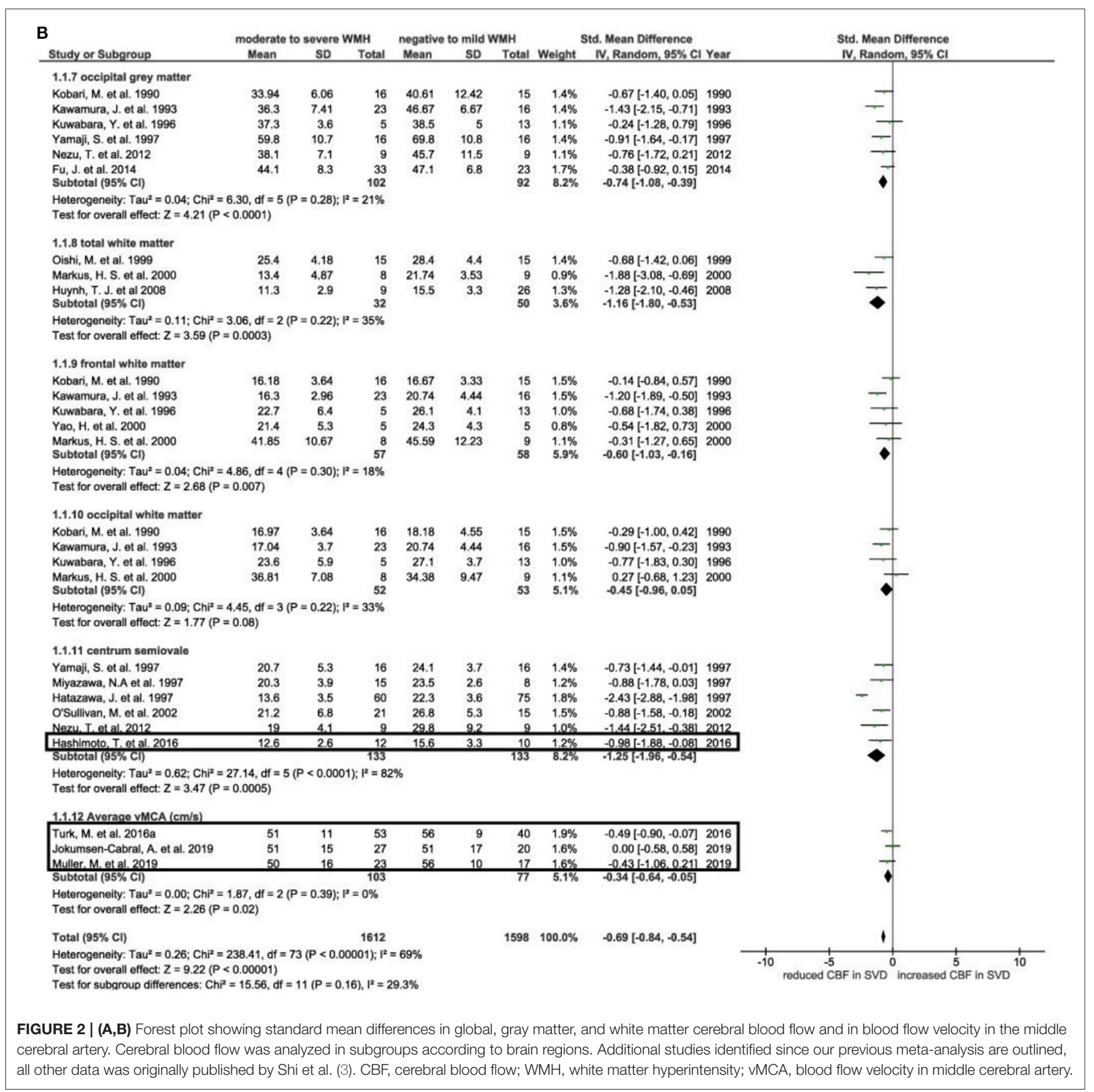

\section{Sensitivity Analyses of Age and Dementia}

As with our previous review (3), the difference in CBF between patients with moderate-to-severe and negative-tomild WMH burden attenuated and became non-significant upon removing studies investigating patients with dementia and further removing non-age matched studies from the updated meta-analysis, apart from mean global CBF and centrum semiovale CBF (Supplementary Figures 3, 4). Mean middle cerebral artery blood flow velocity was not significantly different on comparing individuals with moderate-to-severe and negative-to-mild WMH burden after excluding nonage matched studies. Seven of the ten additional studies were included in the meta-analysis after removing studies which investigated patients with dementia and/or without age-matching; and the studies included in the mean basal ganglia and centrum semiovale categories did not differ, i.e., none of these studies included patients with dementia/without age-matching. 


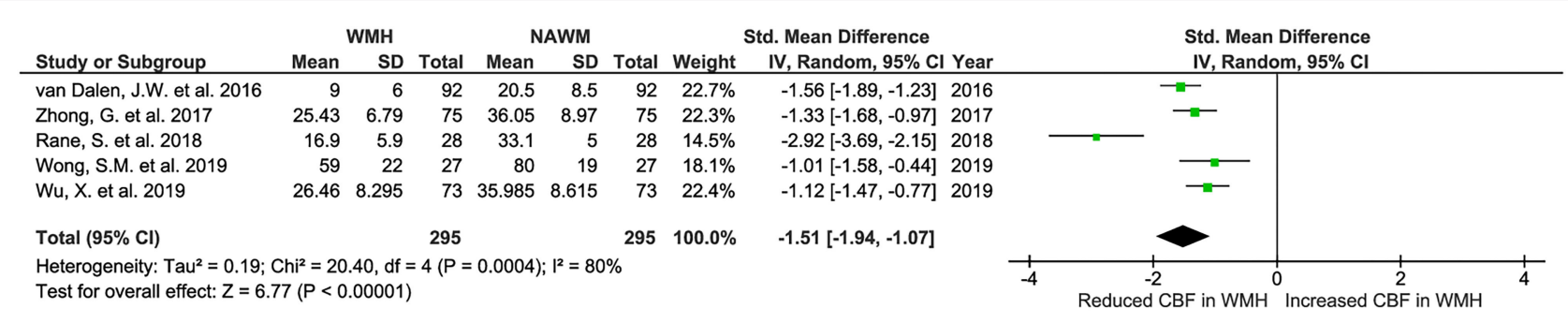

FIGURE 3 | Forest plot of the additional meta-analysis, showing standard mean differences in CBF in WMH compared to NAWM in subjects with moderate to severe WMH burden. WMH, white matter hyperintensity; CBF, cerebral blood flow; NAWM, normal appearing white matter.

\section{Cross-Sectional Meta-Analysis of CBF Difference Between WMH and NAWM}

Additional to the analyses that were possible in 2016, we found sufficient data for meta-analysis in five recent cross-sectional studies reporting CBF values for both WMH and NAWM in subjects with moderate to severe WMH burden $(n=295)$. CBF was lower in WMH compared to NAWM (SMD: $-1.51,95 \%$ CI: $-1.94,-1.07$ ) (Figure 3).

\section{DISCUSSION}

We identified 30 new relevant publications since 2016, providing a total additional sample of 3,396 (cross-sectional $n=2,956$ + longitudinal $n=440$ ). The newly identified cross-sectional studies reported lower CBF in most patients with higher $\mathrm{WMH}$ burden, supporting previous findings (3). In an additional metaanalysis, we confirmed that CBF was also lower in WMH than in NAWM. Longitudinal findings were mixed, the largest studies ( $n$ $=575+252$ ) found baseline global CBF was not associated with $\mathrm{WMH}$ progression (19), as such there is no definite association between low $\mathrm{CBF}$ and increasing $\mathrm{WMH}$ long term. However, regional associations between lower $\mathrm{CBF}$ and $\mathrm{WMH}$ progression, notably in normal-appearing tissue surrounding PVWMH $(n=$ $390+52)(15,38)$ may exist.

Higher WMH burden was also associated with a lower blood flow velocity in the middle cerebral artery, which could be an important contributor to hypoperfusion $(17,35)$. Although blood flow measurements from the middle cerebral artery are less representative of $\mathrm{CBF}$ compared to $\mathrm{CT}$ and MRI-based imaging methods, there is a dynamic relationship between middle cerebral artery blood flow and blood flow in the cerebral small vessels (39). Therefore, flow measurements from the large arteries can be informative of cerebrovascular blood flow (40). An association between impaired hemodynamics in the middle cerebral artery and WMH has previously been established, however there may be a stronger association with higher pulsatility and WMH compared to lower blood flow velocity and WMH (41). One cross-sectional study showed that higher SVD burden was associated with prolonged MTT on CT and DSC (12).

The heterogeneity of study populations can be an important confounder, particularly in terms of age, sex and vascular risk factors. Only a minority (5/11) of cross-sectional studies and none of the longitudinal studies adjusted for cardiovascular risk factors. Cardiovascular risk factors can influence the development and rate of progression of SVD, while also affecting CBF via several mechanisms (42). Hypertension is an established risk factor for WMH that may affect CBF through vascular morphological changes and lumen narrowing, which can contribute to hypoperfusion (43). One cross-sectional study $(n=73)$ found cerebral perfusion was lower in patients with hypertension, with or without $\mathrm{WMH}$, than in healthy controls, though sample size was limited (29). Meanwhile, diabetes has been linked to increased blood viscosity and a reduction in deformability of erythrocytes proportional to the degree of microvascular complications (44), white matter CBF is also lower in patients with type 2 diabetes than in controls (45). As such, controlling for cardiovascular risk factors is crucial in avoiding potential confounds.

\section{Strengths and Limitations}

Strengths of this work include the thorough meta-analysis of regional $\mathrm{CBF}$ variability by $\mathrm{WMH}$ burden, obtained through updating previous work (3). The sample size for studies included in the cross-sectional meta-analysis was enhanced $(n=2,180$, 64/study). We also screened non-English language publications, including a paper published in Russian (29). Limitations of this work include the lack of additional data in some brain subregions, though attempts were made to contact several authors to acquire unpublished data. However, this may also reflect a narrowing focus to key regions based on experimental hypotheses, excluding regions which are less reproducible. The meta-analysis of $\mathrm{CBF}$ variability in WMH and NAWM was limited by the small number of studies available (5), although the average sample size was reasonable ( $n=295$, mean 59/study). Several studies lacked control participants with little to no WMH burden and so could not be included. Longitudinal studies remain very limited and used heterogeneous methods, preventing direct comparison and longitudinal meta-analysis. Follow-up CBF measurements were only reported in 2 longitudinal studies $(18,38)$, thus interpretation of CBF changes over time in association with $\mathrm{WMH}$ progression is limited. However, an increasing use of non-contrast perfusion methods, particularly ASL, should make longitudinal imaging more viable. The role of other SVD 
markers, including lacunes and enlarged perivascular spaces, is also underexplored. Lastly, the accuracy of white matter and WMH perfusion measurements using ASL remains unclear, due to the lower perfusion level and signal-to-noise ratio relative to gray matter. This can be particularly relevant for older patients with cerebrovascular disease, where lower perfusion is more difficult to quantify. However, there are on-going efforts to improve sensitivity of acquisition and analysis techniques (46). Additionally, none of the included ASL studies measured ATT, perhaps because ATT quantification requires a longer acquisition (47).

\section{Interpretation in Relation to CBF and WMH Pathology}

WMH and NAWM CBF variability remains underexplored and more detailed longitudinal studies evaluating subregional changes in tissue $\mathrm{CBF}$ and $\mathrm{WMH}$ progression are needed. $\mathrm{CBF}$ reductions were more severe within $\mathrm{WMH}$ compared to NAWM in patients with moderate-to-severe WMH burden, suggesting WMH represent areas of focal perfusion deficits which are more severe than global hypoperfusion. However, associations with CBF may also be affected by other mechanisms implicated in WMH evolution (48). Two cross-sectional studies suggested that perfusion may be higher with higher $\mathrm{WMH}$ burden in normal-appearing white and some gray matter regions $(6,37)$, but were non-conclusive. Compensatory mechanisms may also affect regional perfusion, with areas of increased perfusion potentially counteracting deficiencies elsewhere (49). However, it is unclear whether compensatory perfusion changes would be sustained if they do occur, and could instead indicate dysfunctional blood flow regulation with worse WMH burden. Replication and more detailed subregional analyses of tissue changes in larger cohorts over time are required. Subtle regional differences in perfusion may also be masked within larger regions (15). The selection of regions of interest should therefore be considered carefully, and may favor finer grained regions, such as stratifying tissue by proximity to existing WMH (50) or other pathological features. We suggest that future studies investigating associations between $\mathrm{WMH}$ and perfusion should include the following key features as a minimum standard: discrimination of flow values in WMH from NAWM, as well as the reporting of vascular risk factors and the inclusion of age-matched controls to avoid these variables confounding the understanding of the relationship between $\mathrm{WMH}$ and $\mathrm{CBF}$.

Different vascular pathologies are thought to underlie PVWMH and DWMH, with PVWMH more likely to result from hemodynamic insufficiency, while DWMH are more likely to be a consequence of SVD (51). Moreover, PVWMH and DWMH have been demonstrated to show different associations with various cardiovascular risk factors, further suggesting different underlying pathologies (52). This highlights the importance of conducting a region-dependent analysis of WMH. Of the 11 cross-sectional studies investigating the association between $\mathrm{WMH}$ and $\mathrm{CBF}$ included in this review, only 3 of these studies provided separate regional analysis for PVWMH and DWMH, while only 1 of the 3 longitudinal studies provided separate data for PVWMH and DWMH. This lack of region-dependent analyses of $\mathrm{WMH}$ limits the interpretation of the association with CBF, given the differences in underlying pathophysiology between PVWMH and DWMH.

\section{Implications for Future Research}

In conclusion, this systematic review and meta-analysis showed that CBF was lower in most gray and white matter regions of the brain with higher $\mathrm{WMH}$ burden and lower in $\mathrm{WMH}$ than normal appearing white matter. Further studies investigating $\mathrm{CBF}$ along with ATT may help give a more comprehensive understanding of causal and consequential effects in WMH development and progression. Longitudinal studies exploring the effect of CBF changes on WMH burden remain limited. Further longitudinal studies are required to better understand the role of perfusion in WMH development and evolution. Future studies should also adjust for key vascular risk factors known to influence CBF, particularly hypertension, and, where relevant, baseline WMH burden.

\section{DATA AVAILABILITY STATEMENT}

The original contributions presented in the study are included in the article/Supplementary Material, further inquiries can be directed to the corresponding author.

\section{AUTHOR CONTRIBUTIONS}

MS and JW conceived the idea of the study. YS and JW designed the search strategy and provided data files from the original literature review. CS did the data search, extracted data, and conducted the statistical analyses and drafted the report and designed the tables and figures. MS cross-checked the data. JW obtained funding and managed the project. All authors revised and approved the manuscript.

\section{FUNDING}

The authors disclosed receipt of the following financial support for the research, authorship and/or publication of this article: NHS Lothian R+D Department (MT); the UK Dementia Research Institute which receives its funding from DRI Ltd, funded by the UK MRC, Alzheimer's Research UK and Alzheimer's Society (MS and JW); the Fondation Leducq Transatlantic Network of Excellence for the Study of Perivascular Spaces in Small Vessel Disease (reference number 16 CVD 05) (MS and JW); and 
European Union Horizon 2020 (project number 666881, SVDs@Target) (MS and JW).

\section{ACKNOWLEDGMENTS}

We would like to thank Tetiana Poliakova for translating the Russian article included in this review and Wenwen $\mathrm{Li}$ and Junfang Zhang for screening a Mandarin

\section{REFERENCES}

1. Wardlaw JM, Smith C, Dichgans M. Small vessel disease: mechanisms and clinical implications. Lancet Neurol. (2019) 18:684-96. doi: 10.1016/S1474-4422(19)30079-1

2. Nasel C, Boubela R, Kalcher K, Moser E. Normalised time-to-peakdistribution curves correlate with cerebral white matter hyperintensities could this improve early diagnosis? J. Cereb. Blood Flow Metab. (2017) 37:444-55. doi: 10.1177/0271678X16629485

3. Shi Y, Thrippleton MJ, Makin SD, Marshall I, Geerlings MI, de Craen AJM, et al. Cerebral blood flow in small vessel disease: a systematic review and meta-analysis. J. Cereb. Blood Flow Metab. (2016) 36:165367. doi: $10.1177 / 0271678 X 16662891$

4. Del CVHM, Ritchie S, Glatz A, Allerhand M, Munoz Maniega S, Gow AJ, et al. Brain iron deposits and lifespan cognitive ability. Age. (2015) 37:100. doi: 10.1007/s11357-015-9837-2

5. Wardlaw JM, Chappell FM, Valdes Hernandez MDC, Makin SDJ, Staals J, Shuler K, et al. White matter hyperintensity reduction and outcomes after minor stroke. Neurology. (2017) 89:1003-10. doi: 10.1212/WNL.0000000000004328

6. van Dalen JW, Mutsaerts H, Nederveen AJ, Vrenken H, Steenwijk MD, Caan MWA, et al. White matter hyperintensity volume and cerebral perfusion in older individuals with hypertension using arterial spin-labeling. AJNR Am. J. Neuroradiol. (2016) 37:1824-30. doi: 10.3174/ajnr.A4828

7. Zhong G, Zhang R, Jiaerken Y, Yu X, Zhou Y, Liu C, et al. Better correlation of cognitive function to white matter integrity than to blood supply in subjects with leukoaraiosis. Front. Aging Neurosci. (2017) 9:185. doi: 10.3389/fnagi.2017.00185

8. Rane S, Koh N, Boord P, Madhyastha T, Askren MK, Jayadev S, et al. Quantitative cerebrovascular pathology in a communitybased cohort of older adults. Neurobiol. Aging. (2018) 65:7785. doi: 10.1016/j.neurobiolaging.2018.01.006

9. Wong SM, Jansen JFA, Zhang CE, Hoff EI, Staals J, van Oostenbrugge $\mathrm{RJ}$, et al. Blood-brain barrier impairment and hypoperfusion are linked in cerebral small vessel disease. Neurology. (2019) 92:e1669-e77. doi: 10.1212/WNL.0000000000007263

10. Wu X, Ge X, Du J, Wang Y, Sun Y, Han X, et al. Characterizing the penumbras of white matter hyperintensities and their associations with cognitive function in patients with subcortical vascular mild cognitive impairment. Front. Neurol. (2019) 10:348. doi: 10.3389/fneur.2019.00348

11. Bailey EL, Smith C, Sudlow CL, Wardlaw JM. Pathology of lacunar ischemic stroke in humans-a systematic review. Brain Pathol. (2012) 22:58391. doi: $10.1111 /$ j.1750-3639.2012.00575.x

12. Arba F, Mair G, Carpenter T, Sakka E, Sandercock PAG, Lindley RI, et al. Cerebral white matter hypoperfusion increases with small-vessel disease burden. Data from the third international stroke trial. J. Stroke Cerebrovasc. Dis. (2017) 26:1506-13. doi: 10.1016/j.jstrokecerebrovasdis.2017. 03.002

13. Staals J, Makin SD, Doubal FN, Dennis MS, Wardlaw JM. Stroke subtype, vascular risk factors, and total MRI brain small-vessel disease burden. Neurology. (2014) 83:1228-34. doi: 10.1212/WNL.0000000000000837

14. Promjunyakul NO, Lahna DL, Kaye JA, Dodge HH, Erten-Lyons D, Rooney WD, et al. Comparison of cerebral blood flow and structural penumbras in relation to white matter hyperintensities: a multi-modal magnetic paper which did not satisfy the inclusion criteria. We thank Dr. Francesca Chappell for providing advice on statistical analysis.

\section{SUPPLEMENTARY MATERIAL}

The Supplementary Material for this article can be found online at: https://www.frontiersin.org/articles/10.3389/fneur. 2021.647848/full\#supplementary-material resonance imaging study. J. Cereb. Blood Flow Metab. (2016) 36:152836. doi: $10.1177 / 0271678 X 16651268$

15. Promjunyakul NO, Dodge HH, Lahna D, Boespflug EL, Kaye JA, Rooney WD, et al. Baseline NAWM structural integrity and CBF predict periventricular WMH expansion over time. Neurology. (2018) 90:e2119e26. doi: 10.1212/WNL.0000000000005684

16. Turk M, Zaletel M, Pretnar Oblak J. Characteristics of cerebral hemodynamics in patients with ischemic leukoaraiosis and new ultrasound indices of ischemic leukoaraiosis. J. Stroke Cerebrovasc. Dis. (2016) 25:97784. doi: 10.1016/j.jstrokecerebrovasdis.2015.12.045

17. Turk M, Zaletel M, Pretnar-Oblak J. Ratio between carotid artery stiffness and blood flow - a new ultrasound index of ischemic leukoaraiosis. Clin. Interv. Aging. (2016) 11:65-71. doi: 10.2147/CIA.S94163

18. Staffaroni AM, Cobigo Y, Elahi FM, Casaletto KB, Walters SM, Wolf A, et al. A longitudinal characterization of perfusion in the aging brain and associations with cognition and neural structure. Hum. Brain Mapp. (2019) 40:3522-33. doi: 10.1002/hbm.24613

19. Nylander R, Fahlstrom M, Rostrup E, Kullberg J, Damangir S, Ahlstrom H, et al. Quantitative and qualitative MRI evaluation of cerebral small vessel disease in an elderly population: a longitudinal study. Acta Radiol. (2018) 59:612-8. doi: 10.1177/0284185117727567

20. Li RR, He YS, Liu M, Nie ZY, Huang LH, Lu Z, et al. Analysis of correlation between cerebral perfusion and KIM score of white matter lesions in patients with Alzheimer's disease. Neuropsychiatr. Dis. Treat. (2019) 15:270514. doi: 10.2147/NDT.S207069

21. Ishibashi M, Kimura N, Aso Y, Matsubara E. Effects of white matter lesions on brain perfusion in patients with mild cognitive impairment. Clin. Neurol. Neurosurg. (2018) 168:7-11. doi: 10.1016/j.clineuro.2018.02.030

22. Shokouhi M, Qiu D, Samman Tahhan A, Quyyumi AA, Hajjar I. Differential associations of diastolic and systolic pressures with cerebral measures in older individuals with mild cognitive impairment. Am. J. Hypertens. (2018) 31:1268-77. doi: 10.1093/ajh/hpy104

23. Benedictus MR, Leeuwis AE, Binnewijzend MA, Kuijer JP, Scheltens P, Barkhof F, et al. Lower cerebral blood flow is associated with faster cognitive decline in Alzheimer's disease. Eur. Radiol. (2017) 27:116975. doi: $10.1007 / \mathrm{s} 00330-016-4450-\mathrm{z}$

24. Shi Y, Thrippleton MJ, Blair GW, Dickie DA, Marshall I, Hamilton I, et al. Small vessel disease is associated with altered cerebrovascular pulsatility but not resting cerebral blood flow. J. Cereb. Blood Flow Metab. (2020) 40:8599. doi: $10.1177 / 0271678 \times 18803956$

25. Muller M, Osterreich M, von Hessling A, Smith RS. Incomplete recovery of cerebral blood flow dynamics in sufficiently treated high blood pressure. J. Hypertens. (2019) 37:372-9. doi: 10.1097/HJH.0000000000001854

26. Kate M, Gioia L, Jeerakathil T, Hill MD, Gould B, McCourt R, et al. Aggressive blood pressure reduction is not associated with decreased perfusion in leukoaraiosis regions in acute intracerebral hemorrhage patients. PLOS ONE. (2019) 14:e0213645. doi: 10.1371/journal.pone.0213645

27. Dolui S, Tisdall D, Vidorreta M, Jacobs DR, Jr., Nasrallah IM, Bryan RN, et al. Characterizing a perfusion-based periventricular small vessel region of interest. Neuroimage Clin. (2019) 23:101897. doi: 10.1016/j.nicl.2019.101897

28. Jokumsen-Cabral A, Aires A, Ferreira S, Azevedo E, Castro P. Primary involvement of neurovascular coupling in cerebral autosomal-dominant arteriopathy with subcortical infarcts and leukoencephalopathy. J. Neurol. (2019) 266:1782-8. doi: 10.1007/s00415-019-09331-y 
29. Parfenov VA, Ostroumova TM, Perepelova EM, Perepelov VA, Kochetkov AI, Ostroumova OD. Brain perfusion, cognitive functions, and vascular age in middle aged patients with essential arterial hypertension. Kardiologiia. (2018) 17:23-31. doi: 10.18087/cardio.2018.5.10117

30. Croall ID, Tozer DJ, Moynihan B, Khan U, O’Brien JT, Morris RG, et al. Effect of standard vs intensive blood pressure control on cerebral blood flow in small vessel disease. The PRESERVE randomized clinical trial. JAMA Neurol. (2018) 75:720-7. doi: 10.1001/jamaneurol.2017.5153

31. Bivard A, Cheng X, Lin L-T, Levi C, Spratt N, Kleinig T, et al. Global white matter hypoperfusion on CT predicts larger infarcts and hemorrhagic transformation after acute ischemia. CNS Neurosci. Ther. (2016) 22:23843. doi: $10.1111 / \mathrm{cns} .12491$

32. Hashimoto T, Yokota C, Koshino K, Shimomura R, Hino T, Moriguchi $\mathrm{T}$, et al. Cerebral blood flow and metabolism associated with cerebral microbleeds in small vessel disease. Ann. Nucl. Med. (2016) 30:494500. doi: 10.1007/s12149-016-1086-7

33. Mark I, Seyedsaadat SM, Benson JC, Kallmes DF, Rabinstein AA, Brinjikji W. Leukoaraiosis and collateral blood flow in stroke patients with anterior circulation large vessel occlusion. J. Neurointerv. Surg. (2020) 12:9425. doi: 10.1136/neurintsurg-2019-015652

34. Kim CM, Alvarado RL, Stephens K, Wey HY, Wang DJJ, Leritz EC, et al. Associations between cerebral blood flow and structural and functional brain imaging measures in individuals with neuropsychologically defined mild cognitive impairment. Neurobiol. Aging. (2020) 86:6474. doi: 10.1016/j.neurobiolaging.2019.10.023

35. Puglisi V, Bramanti A, Lanza G, Cantone M, Vinciguerra L, Pennisi $\mathrm{M}$, et al. Impaired cerebral haemodynamics in vascular depression: insights from transcranial doppler ultrasonography. Front. Psychiatry. (2018) 9:316. doi: 10.3389/fpsyt.2018.00316

36. Bahrani AA, Powell DK, Yu G, Johnson ES, Jicha GA, Smith CD. White matter hyperintensity associations with cerebral blood flow in elderly subjects stratified by cerebrovascular risk. J. Stroke Cerebrovasc. Dis. (2017) 26:77986. doi: 10.1016/j.jstrokecerebrovasdis.2016.10.017

37. Shi L, Miao X, Lou W, Liu K, Abrigo J, Wong A, et al. The spatial associations of cerebral blood flow and spontaneous brain activities with white matter hyperintensities-an exploratory study using multimodal magnetic resonance imaging. Front. Neurol. (2017) 8:593. doi: 10.3389/fneur.2017. 00593

38. ten Dam VH, van den Heuvel DM, de Craen AJ, Bollen EL, Murray HM, Westendorp RG, et al. Decline in total cerebral blood flow is linked with increase in periventricular but not deep white matter hyperintensities. Radiology. (2007) 243:198-203. doi: 10.1148/radiol.2431052111

39. Muller M, Osterreich M, Lakatos L, Hessling AV. Cerebral macro- and microcirculatory blood flow dynamics in successfully treated chronic hypertensive patients with and without white mater lesions. Sci. Rep. (2020) 10:9213. doi: 10.1038/s41598-020-66317-x

40. Purkayastha S, Sorond F. Transcranial Doppler ultrasound: technique and application. Semin. Neurol. (2012) 32:411-20. doi: 10.1055/s-0032-1331812

41. Purkayastha S, Fadar O, Mehregan A, Salat DH, Moscufo N, Meier DS, et al. Impaired cerebrovascular hemodynamics are associated with cerebral white matter damage. J. Cereb. Blood Flow Metab. (2014) 34:22834. doi: $10.1038 /$ jcbfm. 2013.180

42. Ostergaard L, Engedal TS, Moreton F, Hansen MB, Wardlaw JM, Dalkara $\mathrm{T}$, et al. Cerebral small vessel disease: capillary pathways to stroke and cognitive decline. J. Cereb. Blood Flow Metab. (2016) 36:30225. doi: 10.1177/0271678X15606723
43. Glodzik L, Rusinek H, Tsui W, Pirraglia E, Kim HJ, Deshpande A, et al. Different relationship between systolic blood pressure and cerebral perfusion in subjects with and without hypertension. Hypertension. (2019) 73:197205. doi: 10.1161/HYPERTENSIONAHA.118.11233

44. Iadecola C. Neurovascular regulation in the normal brain and in Alzheimer's disease. Nat. Rev. Neurosci. (2004) 5:347-60. doi: 10.1038/nrn1387

45. Shen Y, Zhao B, Yan L, Jann K, Wang G, Wang J, et al. Cerebral hemodynamic and white matter changes of type 2 diabetes revealed by multi-TI arterial spin labeling and double inversion recovery sequence. Front. Neurol. (2017) 8:717. doi: 10.3389/fneur.2017.00717

46. Wu WC, Lin SC, Wang DJ, Chen KL, Li YD. Measurement of cerebral white matter perfusion using pseudocontinuous arterial spin labeling 3T magnetic resonance imaging-an experimental and theoretical investigation of feasibility. PLoS ONE. (2013) 8:e82679. doi: 10.1371/journal.pone.0082679

47. Alsop DC, Detre JA, Golay X, Gunther M, Hendrikse J, Hernandez-Garcia L, et al. Recommended implementation of arterial spin-labeled perfusion MRI for clinical applications: a consensus of the ISMRM perfusion study group and the European consortium for ASL in dementia. Magn. Reson. Med. (2015) 73:102-16. doi: 10.1002/mrm.25197

48. Munoz Maniega S, Chappell FM, Valdes Hernandez MC, Armitage PA, Makin SD, Heye AK, et al. Integrity of normal-appearing white matter: influence of age, visible lesion burden and hypertension in patients with small-vessel disease. J. Cereb. Blood Flow Metab. (2017) 37:64456. doi: 10.1177/0271678X16635657

49. Reuter-Lorenz PA, Cappell KA. Neurocognitive aging and the compensation hypothesis. Curr. Dir. Psychol. Sci. (2008) 17:177-82. doi: 10.1111/j.1467-8721.2008. 00570.x

50. Maniega SM, Valdes Hernandez MC, Clayden JD, Royle NA, Murray $\mathrm{C}$, Morris $\mathrm{Z}$, et al. White matter hyperintensities and normal-appearing white matter integrity in the aging brain. Neurobiol. Aging. (2015) 36:90918. doi: 10.1016/j.neurobiolaging.2014.07.048

51. Kim KW, MacFall JR, Payne ME. Classification of white matter lesions on magnetic resonance imaging in elderly persons. Biol. Psychiatry. (2008) 64:273-80. doi: 10.1016/j.biopsych.2008.03.024

52. Griffanti L, Jenkinson M, Suri S, Zsoldos E, Mahmood A, Filippini N, et al. Classification and characterization of periventricular and deep white matter hyperintensities on MRI: a study in older adults. Neuroimage. (2018) 170:17481. doi: 10.1016/j.neuroimage.2017.03.024

Conflict of Interest: The authors declare that this study received academic grant funding from the UK Dementia Research Institute which receives its funding from DRI Ltd (funded by the UK MRC, Alzheimer's Research UK and Alzheimer's Society), the Fondation Leducq (16 CVD 05), European Union Horizon 2020 (666881, SVDs@Target) and NHS Lothian R+D Department as detailed in the Funding information. The funders were not involved in the study design, collection, analysis, interpretation of data, the writing of this article or the decision to submit it for publication.

Copyright () 2021 Stewart, Stringer, Shi, Thrippleton and Wardlaw. This is an openaccess article distributed under the terms of the Creative Commons Attribution License (CC BY). The use, distribution or reproduction in other forums is permitted, provided the original author(s) and the copyright owner(s) are credited and that the original publication in this journal is cited, in accordance with accepted academic practice. No use, distribution or reproduction is permitted which does not comply with these terms. 